UDC [004.942:519.6]:539.3

A.Yu. Bazhanova, $\mathrm{PhD}$,

M.G. Suryaninov, DEng, Prof.,

G.B. Shotadze

Odessa National Polytechnic University, 1 Shevchenko Ave., 65044 Odessa, Ukraine; e-mail: dedushka27@te.net.ua

\title{
FINITE ELEMENTS MATHEMATICAL MODEL OF GEOMETRIC NONLINEARITY
}

\begin{abstract}
А.Ю. Бажанова, М.Г. Сур'янінов, Г.Б. Шотадзе. Кінцево-елементна математична модель геометричної нелінійності. Розглянуто побудову математичної моделі для врахування геометричної нелінійності в методі кінцевих елементів. Побудована модель дозволяє враховувати зміну матриці жорсткості системи при зміні ії форми. Як об’єкт для порівняння результатів чисельних досліджень на різних моделях з результатами експерименту обрано ресору автомобіля УАЗ - симетричну напівеліптичну ресору, що складається 3 п’ятнадцяти листів. Всі чисельні дослідження виконано у двох CAПР: ANSYS - представник групи важких пакетів — i SolidWorks, який відноситься до середнього рівня. Розроблено пакети автоматизованого проектування, призначені для розширення можливостей існуючих САПР. Аналіз отриманих результатів дозволяє зробити висновок про те, що традиційні моделі нелінійності в ANSYS i SolidWorks дають приблизно однакові результати, які в точці максимуму на 20,6 \% відрізняються від даних натурного експерименту. При використанні запропонованої моделі ця відмінність знижується до 7,95 \%.

Ключові слова: математична модель, геометрична нелінійність, САПР, метод кінцевих елементів, ANSYS, SolidWorks.

A.Yu. Bazhanova, M.G. Suryaninov, G.B. Shotadze. Finite elements mathematical model of geometric nonlinearity. The article considers the construction of a mathematical model for accounting the geometric nonlinearity at finite elements method. The built model takes into account the changes in system stiffness matrix when changing its shape. In quality of an object to compare the results of numerical studies on different models with experimental ones selected is the UAZ automobile spring: symmetric semi-elliptic damper, consisting of fifteen sheets. All numerical studies were performed with two CAD systems: the first one ANSYS, a "heavy" packages representative group, and the SolidWorks, which refers to the average complexity level packages. The results analysis suggests that the traditional nonlinearity model obtained from ANSYS and SolidWorks give approximately the same results at the maximum point of $20,6 \%$ differing from the field experiment data. Using the proposed model, the difference is reduced up to $7,95 \%$

Keywords: mathematical model, geometric nonlinearity, CAD, finite element method, ANSYS, SolidWorks.
\end{abstract}

Introduction. When nonlinear problems solving by finite elements method a significant problem arises. The stiffness matrix depending onto the object displacement the solution obtained using the finite element method (FEM), differs from the real value, and in some cases may even extend beyond the task defining domain (Fig. 1). Therefore it is necessary to apply an iterative approach, gradually increasing the external load from zero to a predetermined value, and stepwise counting the stiffness matrix that simulates the object's real load.

Literature review. Calculations of elastic-dissipative systems, taking into account the geometric and physical nonlinearity are traditionally considered the FEM "weakest link". An extensive research was devoted to that issue. Many authors believe that the non-linearity models used presently, are simply unusable [1...3]. They give proofs with some simple test problems solved by other methods. A comparison with the FEM calculations results shows the complete failure of the latter. And as it is practically impossible to predict the results of non-linear calculation for complex systems their accuracy assessment is only possible with a certain degree of error. In this regard, the new non-linearity models development with a comprehensive CAD test series on different elastic-dissipative systems is of particular importance.

In contrast to the models and methods of contact interaction accounting varied (and sometimes

DOI 10.15276/opu.2.46.2015.25

(C) 2015 The Authors. This is an open access article under the CC BY license (http://creativecommons.org/licenses/by/4.0/). 


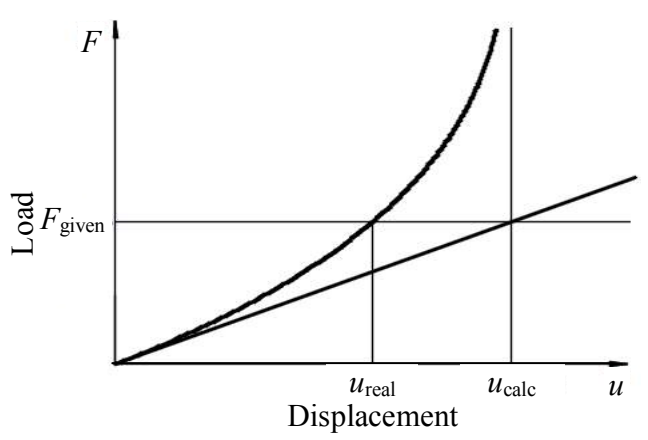

Fig. 1. Difference between the real true and the calculated solutions

quite different) at different CAD systems, the geometric nonlinearity accounting models and methods functionally inherent to $\mathrm{CAD}$, are practically identical. The differences are due to the standard finite elements' types, appropriate to one or the other system and the number of methods used to implement the non-linearity algorithms.

The Aim of the Research therefore refers to constructing a mathematical model for the geometric nonlinearity accounting at FEM.

Main Body. Considering the equation

$$
f(x)=0 .
$$

We write down the generalized iteration formula used to the solution as:

$$
f_{i+1}=f_{i}+\Delta f_{i},
$$

where $f_{i}-i$-th approximate solution of the equation (1) or $i$-th iteration stage's discrepancy.

So we search for a $\Delta f_{i}$ satisfying the $f_{i+1} \rightarrow 0$, then

$$
f_{i}+\Delta f_{i}=0 \text {. }
$$

FEM applied, the (2) get the form

$$
\bar{K} \cdot \vec{u}-\vec{F}=0
$$

where $\bar{K}$ - stiffness matrix;

$\vec{u}$ - points displacement vector;

$\vec{F}$ - external loads vector.

Then, introducing (3) at (2) and reaching the limit,

$$
\left(\bar{K}_{i} \vec{u}_{i}-\vec{F}_{i}\right)+d\left(\bar{K}_{i} \vec{u}_{i}-\vec{F}_{i}\right)=0,
$$

or developing the differential,

$$
\bar{K}_{i} \vec{u}_{i}-\vec{F}_{i}+d \bar{K}_{i} \vec{u}_{i}+\bar{K}_{i} d \vec{u}_{i}-d \vec{F}_{i}=0 .
$$

Therefore,

$$
\bar{K}_{i}\left(\vec{u}_{i}+d \vec{u}_{i}\right)=\left(\vec{F}_{i}+d \vec{F}_{i}\right)-d \bar{K}_{i} \vec{u}_{i} .
$$

The matrix $d \bar{K}_{i}$ is functionally dependent onto displacement increase vector

$$
d \bar{K}_{i}=d \bar{K}_{i}(d \vec{u})
$$

The product term $d \bar{K}_{i} \vec{u}_{i}$ can be transformed as follows:

$$
d \bar{K}_{i}(d \vec{u}) \cdot \vec{u}_{i}=d \bar{K}_{i}(\vec{u}) \cdot d \vec{u}_{i}=\bar{K}_{i}^{*}(\vec{u}) \cdot d \vec{u}_{i} .
$$

Then the formula (4)

$$
\bar{K}_{i} \vec{u}_{i}-\vec{F}_{i}+\bar{K}_{i}^{*}(\vec{u}) \cdot d \vec{u}_{i}+\bar{K}_{i} d \vec{u}_{i}-d \vec{F}_{i}=0,
$$

where $\bar{K}_{i}^{*}(u)-$ stiffness matrix at system reshaping.

Now we consider the rod finite element (Fig. 2, $a$ ) with its linear and angular displacements (Fig. 2, $b$ ).

Identifying members as shown at Fig. 2, we find the similarly positioned element at the conventional model stiffness matrix as

$$
\left(k_{i}\right)_{c}=\left(\cos ^{2} \varphi+2 \frac{u_{e}-u_{b}}{l} \cos \varphi \sin ^{2} \varphi-\frac{v_{e}-v_{b}}{l} \sin \varphi\left(\cos ^{2} \varphi-\sin ^{2} \varphi\right)\right) \frac{E A}{l},
$$


and at the proposed model stiffness matrix as

$$
\begin{gathered}
\left(k_{i}^{*}\right)_{n}=\left(\cos ^{2} \varphi+2 \frac{u_{e}-u_{b}}{l} \cos \varphi \sin ^{2} \varphi-\frac{v_{e}-v_{b}}{l} \sin \varphi\left(\cos ^{2} \varphi-\sin ^{2} \varphi\right)\right) \frac{E A}{l}+ \\
+\left[\sin ^{2} \varphi-\left(\frac{u_{e}-u_{b}}{l} \sin \varphi+\frac{\varphi_{e}-\varphi_{b}}{4}\right) \sin 2 \varphi+\frac{v_{e}-v_{b}}{l} \sin \varphi \cos 2 \varphi\right] \times \\
\times \frac{12 E I G A}{l\left(G A l^{2}+12 f_{s} E I\right)} .
\end{gathered}
$$

Let we consider formulation for the new model stiffness matrix elements $\left(k_{i}^{*}\right)_{n}$.
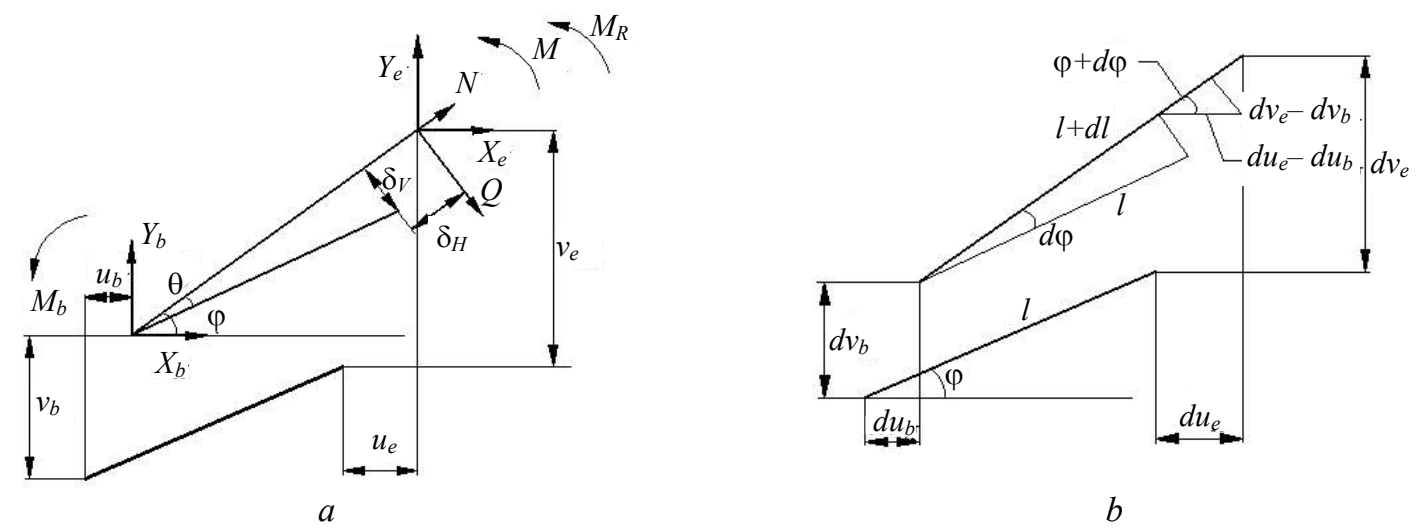

Fig. 2. Rod finite element displacement

We are building the matrix $\bar{K}_{i}^{*}$ for a rod finite element (FE) (Fig. 2, $a$ ). As to other shapes' elements there are no principally distinctive features, exempt for the differences in those FE proper appearance.

Main FEM equation:

$$
\bar{K} \cdot \vec{u}=\vec{F},
$$

where $\vec{u}$ - vector of displacement within global coordinates and consisting of local displacements $u_{i}$;

$\vec{F}$ - vector of load within global coordinates and consisting of load components $f_{i}$;

$\bar{K}$ - global stiffness matrix.

Matrix $\bar{K}-$ the principal component of equation (5), consisting of local stiffness matrixes $\bar{k}$ for all the system's FE

$$
\bar{k}=\bar{a} \bar{b}^{-1} \bar{a}^{-1},
$$

where $\bar{a}-$ matrix of force factors projections;

$\bar{b}-$ compliance matrix accounting the displacementa:

Reciprocal compliance matrix

$$
\begin{aligned}
\bar{a}=\left[\begin{array}{ccc}
-\cos \varphi & -\sin \varphi & 0 \\
-\sin \varphi & \cos \varphi & 0 \\
0 & l & -1 \\
\cos \varphi & \sin \varphi & 0 \\
\sin \varphi & -\cos \varphi & 0 \\
0 & 0 & 1
\end{array}\right] ; \quad \bar{b}=\left[\begin{array}{ccc}
\frac{l}{E A} & 0 & 0 \\
0 & \frac{l^{3}}{3 E I}(1+c) & \frac{l^{2}}{2 E I} \\
0 & \frac{l^{2}}{2 E I} & \frac{l^{2}}{2 E I}
\end{array}\right] ; \\
c=\frac{3 f_{s} E I}{G A l^{2}} .
\end{aligned}
$$


where

$$
\bar{b}^{-1}=\left[\begin{array}{ccc}
\frac{E A}{l} & 0 & 0 \\
0 & \frac{12 E I}{l^{3}} H & \frac{6 E I}{l^{2}} H \\
0 & \frac{12 E I}{l^{3}} H & \frac{4 E I}{l} T
\end{array}\right],
$$

$$
\begin{aligned}
& H=\frac{1}{1+4 c} ; \\
& T=\frac{1+c}{1+4 c} .
\end{aligned}
$$

Introducing (7) and (8) at (6), we get the local stiffness matrix

$$
\bar{k}=\left[\begin{array}{cccccc}
k_{11} & k_{12} & k_{13} & -k_{11} & -k_{12} & k_{16} \\
k_{12} & k_{22} & k_{23} & -k_{12} & -k_{22} & k_{23} \\
k_{13} & k_{23} & k_{33} & -k_{13} & -k_{23} & k_{36} \\
-k_{11} & -k_{12} & -k_{13} & k_{11} & k_{12} & -k_{16} \\
-k_{12} & -k_{22} & -k_{23} & k_{12} & k_{22} & -k_{23} \\
k_{16} & k_{23} & k_{36} & -k_{16} & -k_{23} & k_{33}
\end{array}\right] .
$$

The matrix (9) elements are easily found using (6)...(8):

$$
\begin{gathered}
k_{11}=\frac{E A}{l} \cos ^{2} \varphi+\frac{12 E I}{l^{3}} H \sin ^{2} \varphi, \\
k_{12}=\left(\frac{E A}{l}-\frac{12 E I}{l^{3}} H\right) \sin \varphi \cos \varphi, k_{13}=-\frac{6 E I}{l^{2}} H \sin \varphi, k_{16}=-\frac{3 E I}{l^{2}} H \sin 2 \varphi, \\
k_{36}=\frac{2 E I}{l}(3 H-2 T), k_{22}=\frac{12 E I}{l^{3}} H \cos ^{2} \varphi+\frac{E A}{l} \sin ^{2} \varphi, \\
k_{23}=\frac{6 E I}{l^{2}} H \cos \varphi, k_{33}=\frac{4 E I}{l} T .
\end{gathered}
$$

We shall define the local matrix differential (also easily calculated) with $d \bar{k}$.

Now we consider the finite element displacements during one iteration stage (Fig. 2, $b$ ).

Element elongation

$$
d l=\left(d u_{e}-d u_{b}\right) \cos (\varphi+d \varphi)+\left(d v_{e}-d v_{b}\right) \sin (\varphi+d \varphi) .
$$

Transformation completed we remove the, second smallness order elements, differential factors

$$
\begin{gathered}
d l=-d u_{b} \cos \varphi-d v_{b} \sin \varphi+d u_{e} \cos \varphi+d v_{e} \sin \varphi \\
d \cos \varphi=\frac{\left(u_{e}+d u_{e}\right)-\left(u_{b}+d u_{b}\right)}{l+d l}-\frac{u_{e}-u_{b}}{l}=\frac{l\left(d u_{e}-d u_{b}\right)-d l\left(u_{e}-u_{b}\right)}{l(l+d l)}= \\
=\frac{(l+d l)\left(d u_{e}-d u_{b}\right)-d l\left(u_{e}+d u_{e}-u_{b}-d u_{b}\right)}{l(l+d l)}= \\
=\frac{1}{l}\left(d u_{e}-d u_{b}-\left(-d u_{b} \cos \varphi-d v_{b} \sin \varphi+d u_{e} \cos \varphi+d v_{e} \sin \varphi\right) \cos \varphi\right),
\end{gathered}
$$

or

$$
d \cos \varphi=\frac{1}{l}\left(-d u_{b} \sin ^{2} \varphi+d v_{b} \sin \varphi \cos \varphi+d u_{e} \sin ^{2} \varphi-d v_{e} \sin \varphi \cos \varphi\right) .
$$

By analogy 


$$
\begin{gathered}
d \sin \varphi=\frac{\left(v_{e}+d v_{e}\right)-\left(v_{b}+d v_{b}\right)}{l+d l}-\frac{v_{e}-v_{b}}{l}=\frac{1}{l}\left(d v_{e}-d v_{b}-d l \sin \varphi\right)= \\
=\frac{1}{l}\left(d u_{b} \sin \varphi \cos \varphi-d v_{b} \cos ^{2} \varphi-d u_{e} \sin \varphi \cos \varphi+d v_{e} \cos ^{2} \varphi\right)
\end{gathered}
$$

The equations (10)...(12) can be written down as matrixes

$$
\left[\begin{array}{l}
d l \\
d \cos \varphi \\
d \sin \varphi
\end{array}\right]=\frac{1}{l}\left[\begin{array}{cccccr}
-l \cos \varphi & -l \sin \varphi & 0 & l \cos \varphi & l \sin \varphi & 0 \\
-\sin ^{2} \varphi & \sin \varphi \cos \varphi & 0 & \sin ^{2} \varphi & -\sin \varphi \cos \varphi & 0 \\
\sin \varphi \cos \varphi & -\cos ^{2} \varphi & 0 & -\sin \varphi \cos \varphi & \cos ^{2} \varphi & 0
\end{array}\right] \cdot d \vec{u},
$$

where $d \vec{u}=\left[d u_{b}, d v_{b}, d \varphi_{b}, d u_{e}, d v_{e}, d \varphi_{e}\right]^{T}$ - vector of displacements' increments.

Writing the $d \bar{k}$ with partial derivatives:

$$
d \bar{k}=\sum_{i} \frac{d \bar{k}}{\partial u_{i}} d u_{i}=\frac{d \bar{k}}{\partial u_{b}} d u_{b}+\frac{d \bar{k}}{\partial v_{b}} d v_{b}+\frac{d \bar{k}}{\partial u_{e}} d u_{e}+\frac{d \bar{k}}{\partial v_{e}} d v_{e} .
$$

Therefore, with the account of the agreed notations for matrix's $d \bar{k}$ partial derivatives by displacements

$$
\begin{aligned}
& \frac{\partial \bar{k}}{\partial u_{b}}=\frac{1}{l}\left[\begin{array}{cccccc}
-2 \sin \varphi \cdot k_{12} & \frac{\cos 2 \varphi}{\cos \varphi} k_{12} & -\cos \varphi \cdot k_{16} & 2 \sin \varphi \cdot k_{12} & -\frac{\cos 2 \varphi}{\cos \varphi} k_{12} & -\cos \varphi \cdot k_{16} \\
\frac{\cos 2 \varphi}{\cos \varphi} k_{12} & 2 \sin \varphi \cdot k_{12} & \frac{1+\cos ^{2} \varphi}{\sin \varphi} k_{13} & -\frac{\cos 2 \varphi}{\cos \varphi} k_{12} & -2 \sin \varphi \cdot k_{12} & \sin \varphi \cdot k_{13} \\
\cos \varphi \cdot k_{16} & \frac{1+\cos ^{2} \varphi}{\sin \varphi} k_{13} & -2 l \cdot k_{23} & -\cos \varphi \cdot k_{16} & -\frac{1+\cos ^{2} \varphi}{\sin \varphi} k_{13} & -l \cdot k_{23} \\
2 \sin \varphi \cdot k_{12} & -\frac{\cos 2 \varphi}{\cos \varphi} k_{12} & -\cos \varphi \cdot k_{16} & -2 \sin \varphi \cdot k_{12} & \frac{\cos 2 \varphi}{\cos \varphi} k_{12} & \cos \varphi \cdot k_{16} \\
-\frac{\cos 2 \varphi}{\cos \varphi} k_{12} & -2 \sin \varphi \cdot k_{12} & -\frac{1+\cos { }^{2} \varphi}{\sin \varphi} k_{13} & \frac{\cos 2 \varphi}{\cos \varphi} k_{12} & 2 \sin \varphi \cdot k_{12} & -\sin \varphi \cdot k_{13} \\
-\cos \varphi \cdot k_{16} & \sin \varphi \cdot k_{13} & -l \cdot k_{23} & \cos \varphi \cdot k_{16} & -\sin \varphi \cdot k_{13} & 0
\end{array}\right] \\
& \frac{\partial \bar{k}}{\partial v_{b}}=\frac{1}{l}\left[\begin{array}{cccccc}
2 \cos \varphi \cdot k_{12} & -\frac{\cos 2 \varphi}{\sin \varphi} k_{12} & -\frac{1+\sin ^{2} \varphi}{\sin \varphi} k_{13} & -2 \cos \varphi \cdot k_{12} & \frac{\cos 2 \varphi}{\sin \varphi} k_{12} & \cos \varphi \cdot k_{23} \\
-\frac{\cos 2 \varphi}{\sin \varphi} k_{12} & -2 \cos \varphi \cdot k_{12} & -\cos \varphi \cdot k_{16} & \frac{\cos 2 \varphi}{\sin \varphi} k_{12} & 2 \cos \varphi \cdot k_{12} & \cos \varphi \cdot k_{16} \\
-\frac{1+\sin ^{2} \varphi}{\sin \varphi} k_{13} & -\cos \varphi \cdot k_{16} & 2 l \cdot k_{13} & \frac{1+\sin ^{2} \varphi}{\sin \varphi} k_{13} & \cos \varphi \cdot k_{16} & l \cdot k_{13} \\
-2 \cos \varphi \cdot k_{12} & \frac{\cos 2 \varphi}{\sin \varphi} k_{12} & \frac{1+\sin ^{2} \varphi}{\sin \varphi} k_{13} & 2 \cos \varphi \cdot k_{12} & -\frac{\cos 2 \varphi}{\sin \varphi} k_{12} & -\cos \varphi \cdot k_{23} \\
\frac{\cos 2 \varphi}{\sin \varphi} k_{12} & 2 \cos \varphi \cdot k_{12} & \cos \varphi \cdot k_{16} & -\frac{\cos 2 \varphi}{\sin \varphi} k_{12} & -2 \cos \varphi \cdot k_{12} & -\cos \varphi \cdot k_{16} \\
\cos \varphi \cdot k_{23} & \cos \varphi \cdot k_{16} & l \cdot k_{13} & -\cos \varphi \cdot k_{23} & -\cos \varphi \cdot k_{16} & 0
\end{array}\right] \\
& \frac{\partial k}{\partial u_{e}}=-\frac{\partial k}{\partial u_{b}}, \frac{\partial k}{\partial v_{e}}=-\frac{\partial k}{\partial v_{b}}, \frac{\partial k}{\partial \varphi_{b}}=0, \frac{\partial k}{\partial \varphi_{e}}=0 .
\end{aligned}
$$

The product $d \bar{k}_{i} \vec{u}_{i}$ for a finite element 


$$
d \bar{k}_{i} \vec{u}=d u_{b} \frac{\partial \bar{k}}{\partial u_{b}} \vec{u}+d v_{b} \frac{\partial \bar{k}}{\partial v_{b}} \vec{u}+d u_{e} \frac{\partial \bar{k}}{\partial u_{e}} \vec{u}+d v_{e} \frac{\partial \bar{k}}{\partial v_{e}} \vec{u} .
$$

This sum can be written down in other way, factoring out in parentheses the vector's $\vec{u}$ elements, at that the first member matrix shall be composed of first columns selected from matrixes $\frac{\partial \bar{k}}{\partial u_{b}}$ and $\frac{\partial \bar{k}}{\partial v_{b}}$, the second member matrix - of second columns etc.

Results. In quality of an object to compare the numerical studies results obtained with different models against the experimental results, we selected an UAZ automobile spring, representing a symmetric semi-elliptic damper, that consisting of fifteen plates.

All numerical studies were performed with two CAD systems. Developed are the computer-aided design packages, purposed to enhance the capabilities of existing CAD systems. For the study reasons selected were the CAD ANSYS [4], representative the "heavy" packages group, and the CAD SolidWorks [5], which refers to the average level.

When ANSYS used to develop package applied was the APDL language, specifically designed by ANSYS developers for software extensions. In SolidWorks for the same reason used the macroscenarios allowing an automatic operations' starting.

At the first stage of computer experiments we used the geometric nonlinearity models incorporated by the developers into ANSYS and SolidWorks systems. The main results comparison criterion here was the normal stresses' values that determine the system strength. These stresses are evaluated for ten points uniformly distributed lengthwise the springs. At the second stage we used the proposed nonlinearity model.

Fig. 3 and Fig. 4 show the charts of stresses variation in the springs, built using results calculations with two CADs for different models and identified in the process of full-scale experiment.

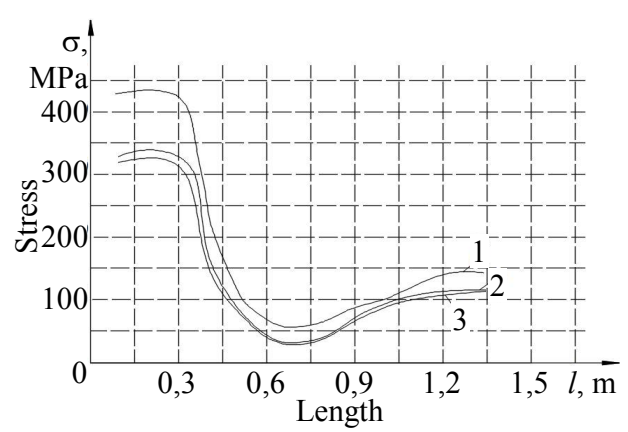

Fig. 3. Geometric nonlinearity conventional model: 1 - experimental, 2 - ANSYS, 3 - SolidWorks

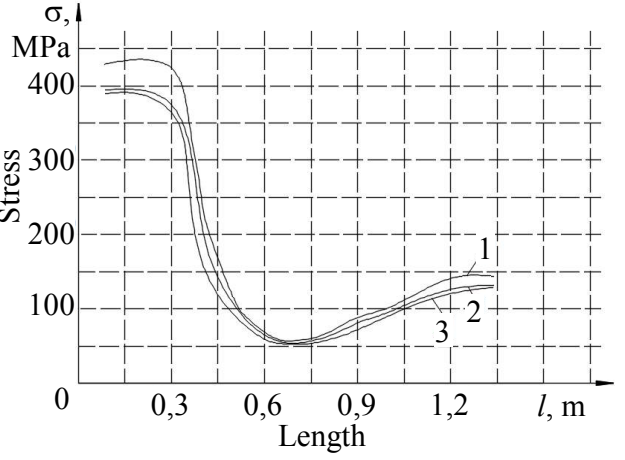

Fig. 4. Geometric nonlinearity new elaborated model: 1 - experimental, 2 - ANSYS, 3 - SolidWorks

Conclusions. The obtained results analysis allows concluding that the traditional nonlinearity model obtained from ANSYS and SolidWorks give approximately the same results at the maximum point of $20,6 \%$ differing from the field experiment data. Using the proposed model, the difference is reduced up to $7,95 \%$

\section{Література}

1. Об использовании метода конечных элементов при решении геометрически нелинейных задач / А.Н. Данилин, Н.Н. Зуев, Д.В. Снеговский, В.И. Шалашилин // САПР и графика. — 2000. — № 4 C. $26-31$.

2. Назаров, Д.И. О «достоверности» расчетов конструкций методом конечных элементов / Д.И. Назаров // САПР и графика. - 2000. - № 7. - С. $23-25$. 
3. Садаков, О.С. О корректности решения геометрически нелинейных задач пакетом МКЭ / О.С. Садаков, А.А. Шапиро // Вестник ЮУрГУ. Серия: Математика, физика, химия. - 2003. № 8(24). - C. 72 - 77 .

4. Дащенко, А.Ф. ANSYS в задачах инженерной механики: монография / А.Ф. Дащенко, Д.В. Лазарева, Н.Г. Сурьянинов; ред. Н.Г. Сурьянинов. - 2-е изд., перераб. и доп. - Харьков: БУРУН и К, 2011. - 504 с.

5. Алямовский, А.A. COSMOSWorks. Основы расчета конструкций на прочность в среде SolidWorks / А.А. Алямовский. — М.: ДМК Пресс, 2010. — 784 с.

\section{References}

1. Danilin, A.N., Zuev, N.N., Snegovsky, D.V. and Shalashilin, V.I. (2000). On the use of the finite element method for solving geometrically nonlinear problems. SAPR and Graphics, 4, 26-31.

2. Nazarov, D.I. (2000). On the "reliability" of structures calculations using finite element method. SAPR and Graphics, 7, 23-25.

3. Sadakov, O.S. and Shapiro, A.A. (2003). About solution correctness of geometrically nonlinear problems with FEM package. Bulletin of the South Ural State University: Mathematics, Physics, Chemistry, 8, 72-77.

4. Dashchenko, A.F., Lazareva, D.V. and Suryaninov, N.G. (2011). ANSYS in Engineering Mechanics Problems ( $2^{\text {nd }}$ Ed.). Kharkiv: BURUN \& Co.

5. Alyamovsky, A.A. (2010). COSMOSWorks. Fundamentals of design of structures for durability in SolidWorks. Moscow: DMK Press.

Received March 15, 2015 